\title{
数控机床几何精度设计指标确定方法研究*
}

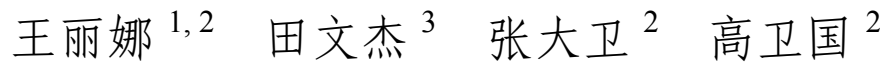

(1. 天津中德应用技术大学机械工程学院 天津 300350 ;

2. 天津大学机械工程学院 天津 300350 ;

3. 天津大学海洋科学与技术学院 天津 300072)

\begin{abstract}
摘要: 针对数控机床几何精度设计过程中整机精度设计指标难于合理确定的问题, 提出一种基于待加工试件公差指标反演机 床刀具工件间相对位姿误差允许变动范围的方法。该方法首先建立试件单一公差项与机床位姿误差之间的约束关系模型, 即 为满足试件单一公差要求, 机床刀具工件间相对位姿误差需要被控制在何种范围内, 然后将所有公差项对应的变动范围求交 集即可得到机床位姿误差的允许变动范围, 并作为整机精度设计的设计指标, 用于机床零部件公差分配。推导了常用公差项 对应的位姿误差变动范围的求解方法, 并以机床精度检验标准中轮廓加工试件为例, 阐述了所提方法的应用过程。
\end{abstract}

关键词: 数控机床; 几何精度; 精度设计; 设计指标; 公差

中图分类号: TH122

\section{Research on Determination Method of Geometric Accuracy Design Index of CNC Machine Tools}

\author{
WANG Lina $^{1,2}$ TIAN Wenjie ${ }^{3}$ ZHANG Dawei ${ }^{2}$ GAO Weiguo ${ }^{2}$ \\ (1. School of Mechanical Engineering, TianjinSino-German University of Applied Sciences, Tianjin 300350; \\ 2. School of Mechanical Engineering, Tianjin University, Tianjin 300350; \\ 3. School of Marine Science and Technology, Tianjin University, Tianjin 300072)
}

\begin{abstract}
Aiming at the problem that the accuracy design index of the machine tool is difficult to be determined reasonably in the geometric precision design process of $\mathrm{CNC}$ machine tools, a method for solving the allowable range of the pose error of the tool relative to the worktable based on the tolerances of the workpiece to be processed is proposed. Firstly, the constraint model between the single tolerance item of the workpiece and the pose error of machine tool is established. That is to say, in order to satisfy the requirement of a single tolerance item, the allowable range of the pose error can be determined. Secondly, the required accuracy design index of the machine tool can be obtained by calculating the intersection of every range corresponding to every single tolerance item, which can then be used for tolerance allocation process. The solution methods of the common tolerance items are derived, and the machining test piece is selected as an example to show the effectiveness of the proposed method.
\end{abstract}

Key words: machine tool; geometric accuracy; accuracy design; design index; tolerance

\section{0 前言}

几何精度是数控机床的重要性能指标 ${ }^{[1]}$ 。为保 障数控机床具备良好的几何精度特性, 通常可采用 如下三种措施: (1) 机床设计阶段, 依据机床精度设 计指标合理分配关键零部件的制造公差; (2) 机床制 造阶段, 严格控制关键零部件的加工与装配误差, 使机床具备良好的基础制造精度; (3) 机床的运行与

* 国家科技重大专项(2018ZX04033001)和国家自然科学基金 (51605324) 资助项目。20190205 收到初稿, 20190605 收到修改稿
使用阶段，通过误差检测与误差补偿等手段进一步 提高机床精度性能 ${ }^{[2-3]}$ 。其中, 设计阶段的公差分配 (又称精度设计)环节是指在保证产品装配技术要求 的前提下，确定各组成环尺寸经济合理的公差的过 程, 是数控机床几何精度保障体系中最基础的一环, 直接影响整机精度品质及制造成本, 目前已越来越 受到机床设计人员的重视。目前的公差分配方法均 将该问题转化为一类以关键零部件公差为设计变 量、以制造成本最低为目标函数、以整机精度指标 为约束条件的最优化问题 ${ }^{[4-9]}$ 。然而, 对于如何合理 确定整机的精度设计指标, 目前尚无妥善的解决方 
案, 仍主要依赖设计人员经验或通过类比同类机床 精度性能指标来确定。例如, 对于机床制造企业而 言, 为使出厂机床产品满足国家标准 ${ }^{[10]}$ 中精加工试 件精度检验要求, 在设计阶段应当如何确定刀具工 件之间位置与姿态误差的允许变动范围, 以指导公 差分配，便属于该类问题。

本文首先简要阐述基于待加工零件公差指标反 演机床刀具工件间相对位姿误差允许变动范围的整 体思路, 然后针对各类常用公差项, 研究其对应的 位姿误差允许变动范围的求解方法, 最后以国标中 轮廓加工试件为例, 给出所提方法的具体应用过程。

\section{1 基本理论}

本文所研究的数控机床几何精度设计指标确定 方法的目的可以概括为, 求解数控机床位置与姿态 误差的允许变动范围, 使得依据此范围设计、制造 出的机床产品, 能够加工出满足特定几何精度要求 的零件。

为此, 记数控机床刀具相对工件的六维位姿误 差为

$$
\boldsymbol{\$}=\left(\begin{array}{c}
\Delta \boldsymbol{r} \\
\Delta \boldsymbol{\theta}
\end{array}\right) \quad \Delta \boldsymbol{r}=\left(\begin{array}{c}
\Delta x \\
\Delta y \\
\Delta z
\end{array}\right) \quad \Delta \boldsymbol{\theta}=\left(\begin{array}{c}
\Delta \alpha \\
\Delta \beta \\
\Delta \gamma
\end{array}\right)
$$

其中, $\Delta \boldsymbol{r} 、 \Delta \boldsymbol{\theta}$ 分别为位置与姿态误差向量。

假定目标零件共有 $n$ 项公差指标要求, 对其中 任意一项公差指标 $T_{i}(i=1,2, \cdots, n)$, 如果可以求得 位姿误差 $\$$ 的变动范围 $\Omega_{1}$, 使得当 $\$ \in \Omega_{\text {时, 公差 }}$ 指标 $T_{i}$ 一定得到满足, 则最终所求位姿误差 $\$$ 的变 动范围为所有 $\Omega_{1}$ 的交集 $\Omega_{1} \cap \Omega_{2} \cap \cdots \cap \Omega_{n}$ 。

因此, 在下一节中将针对常用形状公差、定向 公差、定位公差分别进行研究, 求解各类公差项对 应的机床位姿误差的变动范围的通用表达式。这样, 当一个试件包含多项公差要求时, 只需要对各单一 公差对应的变动范围求交即可。

\section{2 零部件公差与机床位姿误差允许变 动范围间的映射模型}

\section{1 形状公差}

\subsection{1 平面度}

平面度公差记为 $T_{p l a}$ 。平面度公差带是距离为 公差值 $T_{p l a}$ 的两平行平面之间的区域, 这里假定该 公差带相对理想平面呈对称分布。由于端铣和周铣 时对刀具工件相对位姿精度的要求不同, 故分别讨
论分析。

(1) 端铣(被加工平面方向与坐标系方向一致)。

如图 1a 所示, 端铣时, 由于刀具相对工件存在 绕 $x$ 轴、 $y$ 轴的转角误差 $\Delta \alpha 、 \Delta \beta$, 即刀具与工件 被加工表面不垂直, 由此引起的工件表面 $z$ 向加工 误差 $\Delta z=d \Delta \alpha$ 或 $\Delta z=d \Delta \beta$ (其中 $d$ 为刀具直径)。当 刀具端面尺寸 (直径 $d$ )较小, 或者对机床精度要求 不高时, 可忽略该部分影响。刀尖点 $x$ 向、 $y$ 向位 置误差 $\Delta x 、 \Delta y$ 以及刀具绕 $z$ 轴的转角误差 $\Delta \gamma$ 对平 面度没有影响, 故不必考虑。因此, 只需满足刀尖 点 $z$ 向位置误差变动量小于平面度公差值, 即

$$
|\Delta z| \leqslant \frac{T_{p l a}}{2}
$$

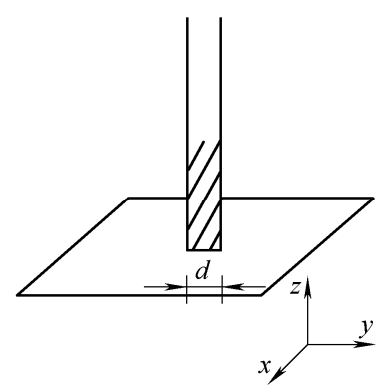

(a) 端铣 (特殊)

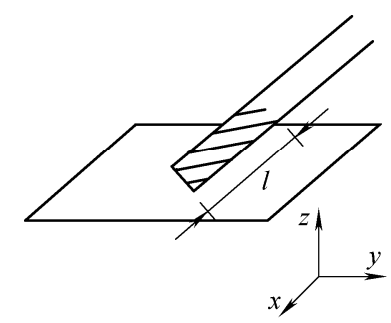

(c) 周铣 (特殊)

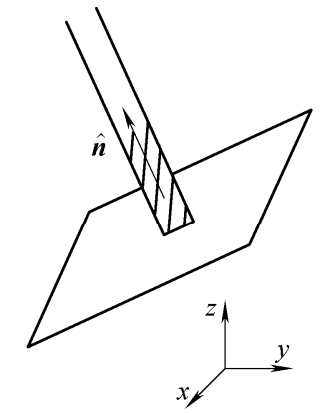

(b) 端铣 (一般)

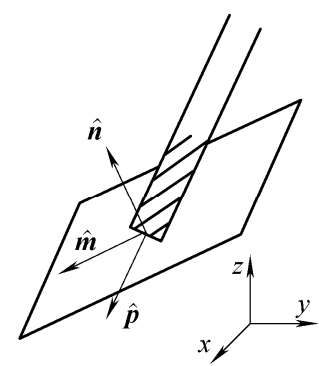

(d) 周铣 (一般 )
图 1 平面度公差

(2) 端铣(被加工平面为空间任意方向)。

如图 $1 \mathrm{~b}$ 所示, 令被加工平面的方程为 $A x+B y+C z=0$, 由于平面度仅对平面形状起到约 束作用, 并未限制其空间位置, 故方程中常数项设 为 0 , 即假定平面经过坐标原点, 则平面法向量可以 表示为 $\boldsymbol{n}=\left(\begin{array}{lll}A & B & C\end{array}\right)^{\mathrm{T}}$, 单位法向量为 $\hat{\boldsymbol{n}}=\boldsymbol{n} /\|\boldsymbol{n}\|$ 。

为保证平面度要求, 刀尖点到理想平面的距离 $d$ 必须小于 $T_{p l a} / 2$ 。这里特别强调的是, $\$$ 中各元素 单独起作用与同时起作用时, $d$ 都必须满足要求, 两者缺一不可。本文中称各元素单独起作用时的约 束不等式为独立约束条件, 各元素同时起作用时的 约束不等式为综合约束条件。

独立约束条件。以刀尖点 $x$ 向位置误差 $\Delta x$ 为 例, $\Delta x$ 的独立约束条件可以表述为: 由 $\Delta x$ 引起的 平面度公差满足设计要求, 即该误差在平面法线方 
向 $\hat{\boldsymbol{n}}$ 上的投影不超过给定平面度公差 $T_{p l a}$ 的一半。

$$
|\hat{\boldsymbol{n}} \cdot \lambda|=\left|\hat{\boldsymbol{n}}^{\mathrm{T}} \lambda\right| \leqslant \frac{T_{p l a}}{2}
$$

其中, $\lambda=\left(\begin{array}{lll}\Delta x & 0 & 0\end{array}\right)^{\mathrm{T}}$ 表示只保留 $x$ 向分量的位置 误差向量 $\Delta \boldsymbol{r}$ 。

分别考虑 $\Delta \boldsymbol{r}$ 的三个分量, 将独立约束条件写成 统一的表达形式如下

$$
\left|\hat{\boldsymbol{n}}^{\mathrm{T}} \lambda\right| \leqslant \frac{T_{p l a}}{2} \quad \lambda=\left(\begin{array}{c}
\Delta x \\
0 \\
0
\end{array}\right),\left(\begin{array}{c}
0 \\
\Delta y \\
0
\end{array}\right),\left(\begin{array}{c}
0 \\
0 \\
\Delta z
\end{array}\right)
$$

综合约束条件。刀具相对工件的位置误差向量 $\Delta \boldsymbol{r}$ 的各个分量同时起作用时, $\Delta \boldsymbol{r}$ 在平面法线方向 $\hat{\boldsymbol{n}}$ 上的投影满足平面度公差要求, 即

$$
\left|\hat{\boldsymbol{n}}^{\mathrm{T}} \Delta \boldsymbol{r}\right| \leqslant \frac{T_{p l a}}{2}
$$

(3) 周铣(被加工平面方向与坐标系方向一致)。

如图 1c 所示, 周铣且切削宽度为 $l$ 时, 除了刀 尖点 $z$ 向位置误差 $\Delta z$ 之外, 刀具绕 $y$ 轴的转角误差 $\Delta \beta$ 也会在被加工平面的 $\mathrm{z}$ 方向上引起大小为 $l \Delta \beta$ 的加工误差。

因此, 当 $\Delta z$ 与 $\Delta \beta$ 分别单独起作用时所引起的 $z$ 向加工误差应分别满足平面度公差要求, 即独立 约束条件为

$$
|\Delta z| \leqslant \frac{T_{p l a}}{2}, \quad|I \Delta \beta| \leqslant \frac{T_{p l a}}{2}
$$

当 $\Delta z$ 与 $\Delta \beta$ 同时起作用时所引起的 $\mathrm{z}$ 向加工误 差应满足平面度公差要求, 即综合约束条件为

$$
|\Delta z+l \Delta \beta| \leqslant \frac{T_{p l a}}{2}
$$

(4) 周铣(被加工平面为空间任意方向)。

令被加工平面的方程为 $A x+B y+C z=0$, 刀具 轴线方向的单位向量为 $\hat{\boldsymbol{p}}$, 则切削进给方向单位向 量 $\hat{\boldsymbol{m}}=\hat{\boldsymbol{p}} \times \hat{\boldsymbol{n}}$, 如图 1d 所示。

首先考虑单项位置、姿态误差单独起作用时所 需满足的独立约束条件。位置误差的独立约束条件 与前述情况(2)完全一致, 只需各项位置误差在平面 法线方向 $\hat{\boldsymbol{n}}$ 上的投影不超过给定平面度公差 $T_{p l a}$ 的 一半，即

$$
\left|\hat{\boldsymbol{n}}^{\mathrm{T}} \lambda\right| \leqslant \frac{T_{p l a}}{2} \quad \lambda=\left(\begin{array}{c}
\Delta x \\
0 \\
0
\end{array}\right),\left(\begin{array}{c}
0 \\
\Delta y \\
0
\end{array}\right),\left(\begin{array}{c}
0 \\
0 \\
\Delta \mathrm{z}
\end{array}\right)
$$

刀具姿态误差在 $\hat{\boldsymbol{p}} 、 \hat{\boldsymbol{n}}$ 方向上的投影对平面度 误差无影响, 即刀具绕 $\hat{\boldsymbol{p}} 、 \hat{\boldsymbol{n}}$ 的转角误差不影响被 加工平面的平面度。而刀具绕 $\hat{\boldsymbol{m}}$ 的转角误差对平面
度的影响与切削宽度 $l$ 相关, 以姿态误差 $\Delta \alpha$ 为例, 其在 $\hat{\boldsymbol{m}}$ 方向的分量所引起的平面度误差需满足

$$
|l \hat{\boldsymbol{m}} \cdot \boldsymbol{\tau}|=\left|\hat{\boldsymbol{m}}^{\mathrm{T}} \boldsymbol{\tau}\right| \leqslant \frac{T_{p l a}}{2}
$$

其中, $\boldsymbol{\tau}=\left(\begin{array}{lll}\Delta \alpha & 0 & 0\end{array}\right)^{\mathrm{T}}$ 表示只保留 $x$ 向分量的姿态 误差向量 $\Delta \boldsymbol{\theta}$ 。

分别考虑 $\Delta \boldsymbol{\theta}$ 的三个分量, 将姿态误差独立约束 条件写成统一的表达形式如下

$$
\left|l \hat{\boldsymbol{m}}^{\mathrm{T}} \boldsymbol{\tau}\right| \leqslant \frac{T_{p l a}}{2}, \text { 其中 } \boldsymbol{\tau}=\left(\begin{array}{c}
\Delta \alpha \\
0 \\
0
\end{array}\right),\left(\begin{array}{c}
0 \\
\Delta \beta \\
0
\end{array}\right),\left(\begin{array}{c}
0 \\
0 \\
\Delta \gamma
\end{array}\right)
$$

综合约束条件为所有误差项同时起作用时所引 起的平面度误差满足设计要求, 即

$$
\left|\hat{\boldsymbol{n}}^{\mathrm{T}} \Delta \boldsymbol{r}+l \hat{\boldsymbol{m}}^{\mathrm{T}} \Delta \boldsymbol{\theta}\right| \leqslant \frac{T_{p l a}}{2}
$$

\subsection{2 直线度}

直线度公差记为 $T_{s t r}$ 。可分为两种情况讨论。

(1) 给定方向的直线度(图 2a)。

给定方向的单位矢量若为 $\hat{\boldsymbol{n}}$, 可以等价为平面 度 $T_{\text {pla }}=T_{\text {str }}$ 的形式。

(2) 未给定方向的直线度(图 2b)。

未给定方向的直线度一般用于圆柱面轴线, 可 等价为圆柱度 $T_{c y l}=T_{s t r}$ 的形式, 详见圆柱度一节。

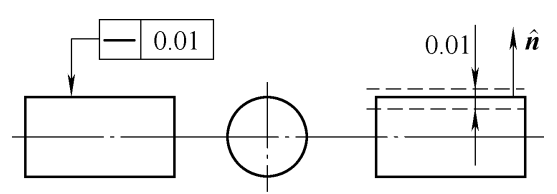

(a) 给定方向

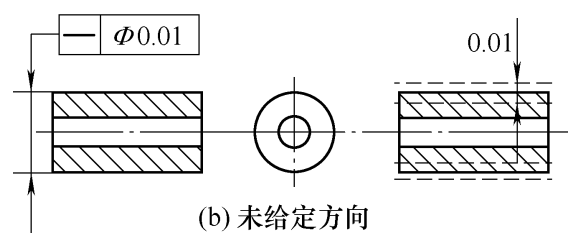

图 2 直线度公差

\subsection{3 圆度}

圆度公差记为 $T_{\text {cir }}$ 。圆度公差带即为在所研究 截面上的圆环区域, 圆环中心圆的半径为理想半 径 $r$, 圆环的径向宽度为公差值 $T_{\text {cir }}$ 。圆度公差一 般标注在圆柱面或圆雉面上: 若为圆柱面, 当圆柱 的轴向尺寸较大时, 圆度公差可转化为圆柱度公 差, 因而本节讨论的圆度误差认为是圆柱面轴向 尺寸较小的情况, 即刀具的转角误差可以忽略的 情况; 若为圆雉面, 可以将圆雉面看成由若干个 半径随轴向位置变化的圆组合而成, 因而同样可以 忽略刀具的转角误差。 
(1) 圆位于坐标平面内(图 3a)。

加工过程中刀尖点必须落于图示公差带范围 内, 当设计变量单独、同时起作用时, 分别得到设 计变量的变动范围与公差约束条件如下。

独立约束条件为

$$
|\Delta x| \leqslant \frac{T_{\text {cir }}}{2},|\Delta y| \leqslant \frac{T_{\text {cir }}}{2}
$$

综合约束条件为: 当刀尖点位置误差 $\Delta x 、 \Delta y$ 同 时起作用时, 刀尖点的实际位置与理论圆心的距离为 $\sqrt{(x+\Delta x)^{2}+(y+\Delta y)^{2}}$, 其中 $x=r \cos \theta 、 y=r \sin \theta$ 为刀尖点理论位置的坐标, 参数 $\theta \in[0,2 \pi)$, 半径 方向的加工误差需控制在圆度公差值的一半以 内, 即

$$
\left|\sqrt{(x+\Delta x)^{2}+(y+\Delta y)^{2}}-r\right| \leqslant \frac{T_{\text {cir }}}{2}
$$

将式(2)展开, 并略去高阶无穷小项(因为公差值 相比半径 $r$ 非常小), 化简得

$$
\sqrt{\Delta x^{2}+\Delta y^{2}} \leqslant \frac{T_{c i r}}{2}
$$

由式(3)可以看出, 圆度公差值的约束条件与半 径 $r$ 无关。

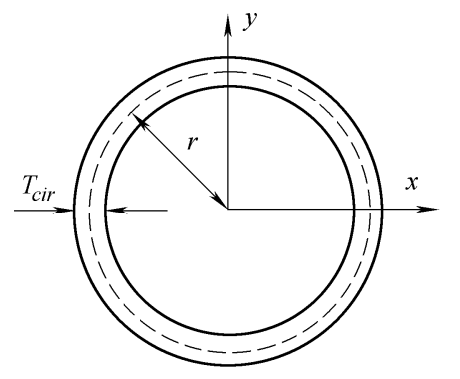

(a) 特殊

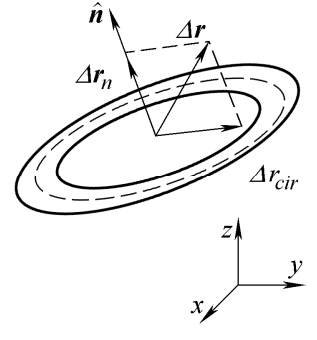

(b) 一般
图 3 圆度公差

(2) 圆位于空间任意平面内(图 3b)。

与平面度公差分析类似, 令圆所在平面方程为 $A x+B y+C z=0$, 其法向量为 $\boldsymbol{n}=\left(\begin{array}{lll}A & B & C\end{array}\right)^{\mathrm{T}}$, 单 位法向量为 $\hat{\boldsymbol{n}}=\boldsymbol{n} /\|\boldsymbol{n}\|$ 。刀尖点位置误差向量 $\Delta \boldsymbol{r}$ 在法 线方向 $\hat{\boldsymbol{n}}$ 的分量 $\Delta \boldsymbol{r}_{n}$ 对圆度误差无影响, 在圆平面 内的分量 $\Delta r_{c i r}$ 对圆度误差的影响与情况(1)类似, 需 满足同样的约束条件。 $\Delta r$ 可以表示为 $\left(\hat{\boldsymbol{n}}^{\mathrm{T}} \lambda\right) \hat{\boldsymbol{n}}, \hat{\boldsymbol{n}}^{\mathrm{T}} \lambda$ 表示模的大小, $\hat{\boldsymbol{n}}$ 表示向量方向, $\Delta \boldsymbol{r}_{\text {cir }}$ 可通过 $\Delta \boldsymbol{r}$ 与 $\Delta \boldsymbol{r}_{n}$ 做差得到。故

独立约束条件为

$$
\left\|\lambda-\left(\hat{\boldsymbol{n}}^{\mathrm{T}} \lambda\right) \hat{\boldsymbol{n}}\right\| \leqslant \frac{T_{\text {cir }}}{2} \quad \lambda=\left(\begin{array}{c}
\Delta x \\
0 \\
0
\end{array}\right),\left(\begin{array}{c}
0 \\
\Delta y \\
0
\end{array}\right),\left(\begin{array}{c}
0 \\
0 \\
\Delta z
\end{array}\right)
$$

综合约束条件为

$$
\left\|\Delta \boldsymbol{r}_{\text {cir }}\right\|=\left\|\Delta \boldsymbol{r}-\Delta \boldsymbol{r}_{n}\right\|=\left\|\Delta \boldsymbol{r}-\left(\hat{\boldsymbol{n}}^{\mathrm{T}} \Delta \boldsymbol{r}\right) \hat{\boldsymbol{n}}\right\| \leqslant \frac{T_{\text {cir }}}{2}
$$

\subsection{4 圆柱度}

圆柱度是半径差为公差值 $T_{c y l}$ 的两同心圆柱面 之间的区域。求解方法与圆度类似, 这里直接给出 结果。

(1) 圆柱轴线与坐标轴同向(图 4a)。

独立约束条件为

$$
|\Delta \alpha| \leqslant \frac{T_{c y l}}{2 l_{c y l}}, \quad|\Delta \beta| \leqslant \frac{T_{c y l}}{2 l_{c y l}}, \quad|\Delta x| \leqslant \frac{T_{c y l}}{2}, \quad|\Delta y| \leqslant \frac{T_{c y l}}{2}
$$

综合约束条件为

$$
\left|\sqrt{(x+z \Delta \beta+\Delta x)^{2}+(y+z \Delta \alpha+\Delta y)^{2}}-r\right| \leqslant \frac{T_{c y l}}{2}
$$

式中, $x=r \cos \theta, y=r \sin \theta$, 中间变量 $\theta \in[0,2 \pi)$, 且 $z \in\left[0, l_{c y l}\right]$ 。式(4)化简后为

$$
\sqrt{\left(l_{c y l} \Delta \beta+\Delta x\right)^{2}+\left(l_{c y l} \Delta \alpha+\Delta y\right)^{2}} \leqslant \frac{T_{c y l}}{2}
$$

(2) 圆柱轴线为空间任意方向(图 4b)。

设圆柱轴线方向单位向量为 $\hat{\boldsymbol{n}}$, 在圆柱任一截 面内找到互相垂直的一对单位向量, 分别记为 $\hat{\boldsymbol{n}}_{x}$ 、 $\hat{\boldsymbol{n}}_{y}$, 将 $\Delta \boldsymbol{r} 、 \Delta \boldsymbol{\theta}$ 投影到 $\hat{\boldsymbol{n}}_{x} 、 \hat{\boldsymbol{n}}_{y}$ 方向, 投影向量分 别记为 $\Delta \boldsymbol{r}_{x} 、 \Delta \boldsymbol{r}_{y} 、 \Delta \boldsymbol{\theta}_{x} 、 \Delta \boldsymbol{\theta}_{\boldsymbol{y}}$, 则问题转化为情况 (1)的形式。

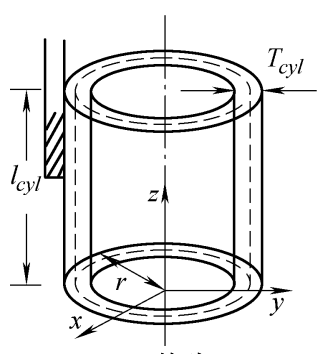

(a) 特殊

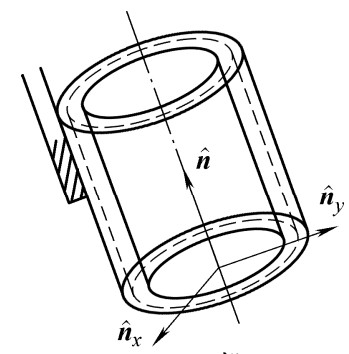

(b) 一般
图 4 圆柱度公差

独立约束条件为

$$
\begin{gathered}
\left\|\Delta \boldsymbol{r}_{x}\right\|=\left|\hat{\boldsymbol{n}}_{x}^{\mathrm{T}} \Delta \boldsymbol{r}\right| \leqslant \frac{T_{c y l}}{2}, \quad\left\|\Delta \boldsymbol{r}_{y}\right\|=\left|\hat{\boldsymbol{n}}_{y}^{\mathrm{T}} \Delta \boldsymbol{r}\right| \leqslant \frac{T_{c y l}}{2} \\
\left\|\Delta \boldsymbol{\theta}_{x}\right\|=\left|\hat{\boldsymbol{n}}_{x}^{\mathrm{T}} \Delta \boldsymbol{\theta}\right| \leqslant \frac{T_{c y l}}{2 l_{c y l}}, \quad\left\|\Delta \boldsymbol{\theta}_{y}\right\|=\left|\hat{\boldsymbol{n}}_{y}^{\mathrm{T}} \Delta \boldsymbol{\theta}\right| \leqslant \frac{T_{c y l}}{2 l_{c y l}}
\end{gathered}
$$

综合约束条件为

$$
\sqrt{\left(l_{c y l} \hat{\boldsymbol{n}}_{y}^{\mathrm{T}} \Delta \boldsymbol{\theta}+\hat{\boldsymbol{n}}_{x}^{\mathrm{T}} \Delta \boldsymbol{r}\right)^{2}+\left(l_{c y l} \hat{\boldsymbol{n}}_{x}^{\mathrm{T}} \Delta \boldsymbol{\theta}+\hat{\boldsymbol{n}}_{y}^{\mathrm{T}} \Delta \boldsymbol{r}\right)^{2}} \leqslant \frac{T_{c y l}}{2}
$$

\section{2 定向公差}

\subsection{1 平行度}

平行度公差记为 $T_{p a r}$ 。依据基准要素的不同, 可分为四种情况讨论。 
(1) 面对面的平行度(图 5a)。

可转化为一个平面相对于另一基准平面的平面 度的问题。

(2) 线对面的平行度(图 5b)。

可转化为轴线在任意方向上的直线度问题, 进 而转化为对应圆柱面的圆柱度的问题。

(3) 面对线的平行度(图 5c)。

可转化为被约束平面在指定方向上的平面度的 问题。

(4) 线对线的平行度(图 5d)。

可转化为被约束直线在任意方向上的直线度问 题, 进而转化为轴线所对应圆柱面的圆柱度的问题。

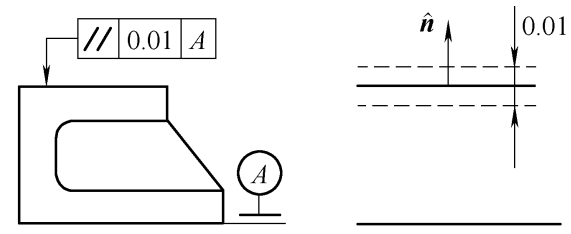

(a) 面对面
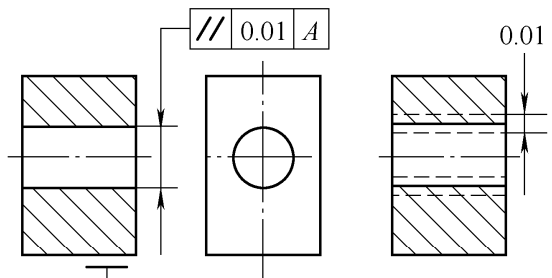

(A)

(b) 线对面
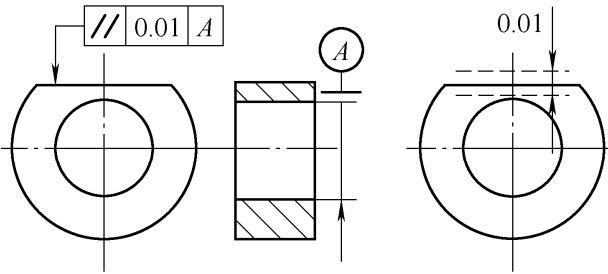

(c) 面对线
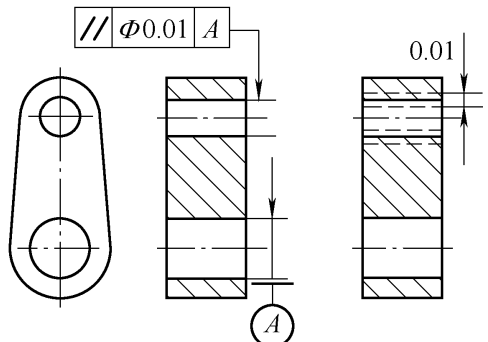

(d) 线对线

图 5 平行度公差

\subsection{2 垂直度}

垂直度公差记为 $T_{p e r}$ 。依据基准要素的不同, 可分为四类讨论。

(1) 面对面的垂直度(图 6a)。

可转化为平面在给定方向 $\hat{\boldsymbol{n}}$ 上的平面度问题。

(2) 面对线的垂直度(图 6b)。

可转化为平面在给定方向 $\hat{\boldsymbol{n}}$ 上的平面度问题。
(3) 线对线的垂直度(图 6c)。 可转化为空间直线直线度的问题。

(4) 线对面的垂直度(图 6d)。

可转化为轴线所对应圆柱面圆柱度的问题。

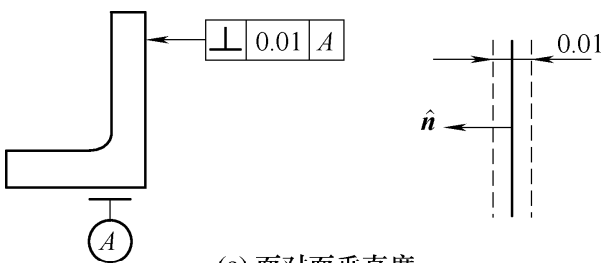

(a) 面对面开直度

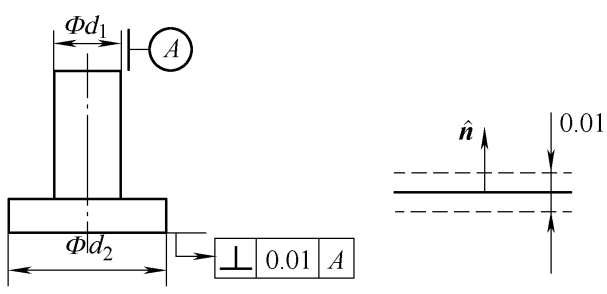

(b) 面对线垂直度

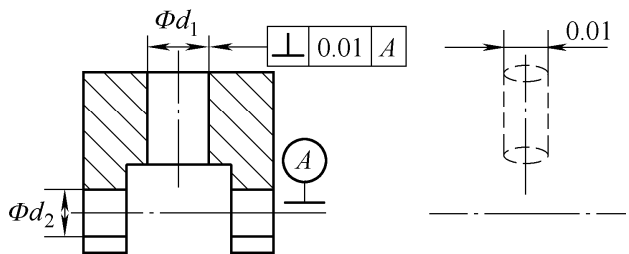

(c) 线对线垂直度

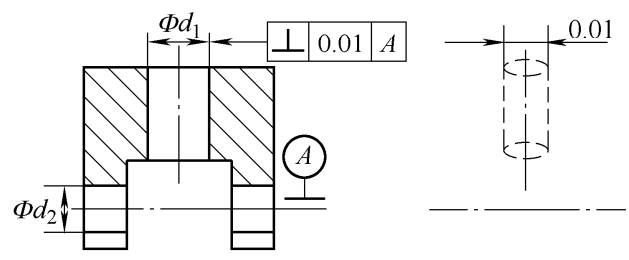

(d) 线对面垂直度

图 6 垂直度公差

\section{2 .3 倾斜度}

倾斜度公差记为 $T_{\text {ang }}$ 。依据基准要素的不同进 行分类讨论。面对线、面对面、线对线的倾斜度示 意图分别如图 7a $\sim 7 \mathrm{c}$ 所示, 均可转化为指定方向上 平面度的问题。

\section{3 定位公差}

\subsection{1 同轴度}

同轴度公差记为 $T_{\text {con }}$ 。如图 8 所示, 同轴度可 首先转化为被约束圆柱面轴线的直线度，进而转化 为圆柱面的圆柱度进行求解。

\subsection{2 位置度}

位置度公差记为 $T_{p o s}$ 。位置度通常指孔的轴线 的位置度, 可首先转化为轴线的直线度, 进而转化 为孔的圆柱度进行求解，具体求解方法详见 3.1 节 算例中公差项(13)的处理方法。 

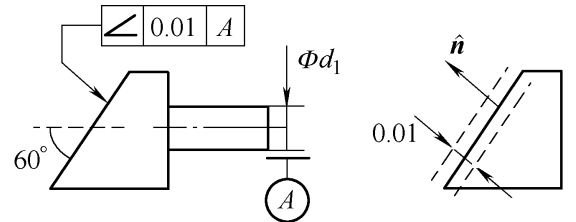

(a) 面对线倾斜度
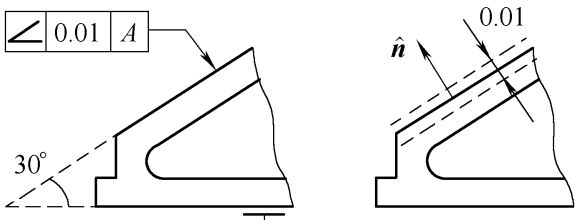

(A)

(b) 面对线倾斜度
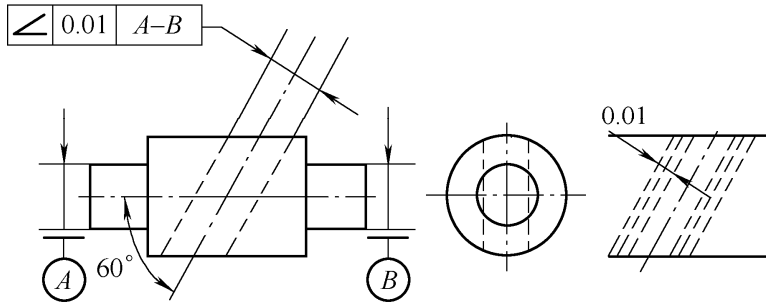

(c) 线对线倾斜度

图 7 倾斜度公差

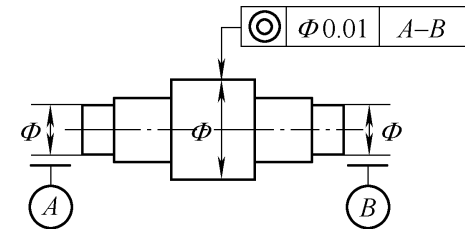

图 8 同轴度公差

\section{3 算例}

本节以国家标准 GBT 20957.7-2007 $7^{[10]}$ 中大规 格轮廓加工试件为例, 研究为满足试件的 13 项公 差要求(图 9), 机床刀具、工件间相对位姿误差必 须满足怎样的变动范围, 以便为机床的精度设计 工作提供设计依据与设计指标。第 3.1 节针对每一 项公差要求, 利用第 2 节中所述方法开展具体的 分析。为描述简洁, 本节中公差项的单位“"mm”均 省略不写。

\section{1 单项公差分析}

(1) 中心孔圆柱度。

公差要求为 $T_{c y l}=0.010$ 。

独立约束条件为

$$
|\Delta \alpha| \leqslant \frac{T_{c y l}}{2 l_{C}}, \quad|\Delta \beta| \leqslant \frac{T_{c y l}}{2 l_{C}}, \quad|\Delta x| \leqslant \frac{T_{c y l}}{2}, \quad|\Delta y| \leqslant \frac{T_{c y l}}{2}
$$

综合约束条件为

$$
\sqrt{\left(l_{C} \Delta \beta+\Delta x\right)^{2}+\left(l_{C} \Delta \alpha+\Delta y\right)^{2}} \leqslant \frac{T_{c y l}}{2}
$$

其中, $l_{C}$ 为中心孔轴向长度。

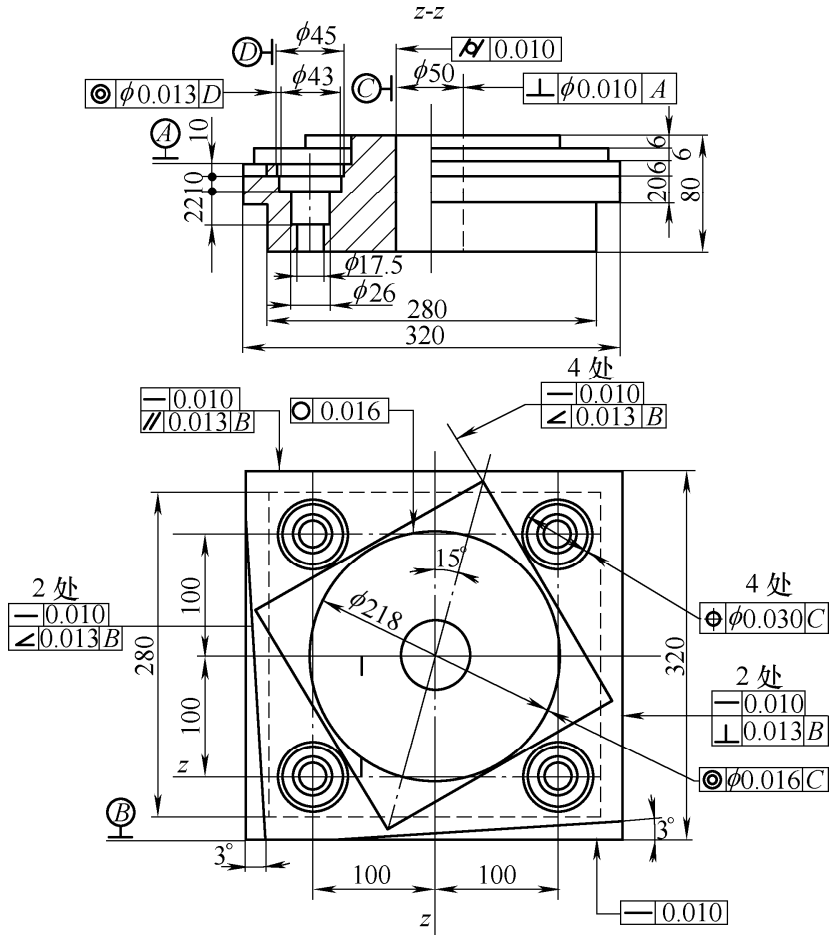

图 9 国标精度检验试件公差要求

(2) 中心孔轴线对基准 $A$ 的垂直度。

公差要求为 $T_{p e r, C}=0.010$, 角标 $C$ 表示基准 $C$ 。 该项垂直度属于线对面的垂直度, 可先转化为轴线 的直线度，进而转化为中心孔圆柱度来考虑。

独立约束条件为

$|\Delta \alpha| \leqslant \frac{T_{p e r, C}}{2 l_{C}},|\Delta \beta| \leqslant \frac{T_{p e r, C}}{2 l_{C}},|\Delta x| \leqslant \frac{T_{p e r, C}}{2},|\Delta y| \leqslant \frac{T_{p e r, C}}{2}$

综合约束条件为

$$
\sqrt{\left(l_{C} \Delta \beta+\Delta x\right)^{2}+\left(l_{C} \Delta \alpha+\Delta y\right)^{2}} \leqslant \frac{T_{p e r, C}}{2}
$$

(3) 正四方形边的直线度。

公差要求为 $T_{\text {str }, \text { squ }}=0.010$ 。转化为直线所在平 面的平面度问题进行求解。

独立约束条件为

$$
|\Delta x| \leqslant \frac{T_{\text {str }, \text { squ }}}{2}, \quad|\Delta y| \leqslant \frac{T_{\text {str,squ }}}{2}
$$

(4) 正四方形相邻边对基准 B 的垂直度。

公差要求为 $T_{p e r, B}=0.013$ 。转化为直线所在平 面相对于基准平面的平面度的问题进行求解。

独立约束条件为

$$
|\Delta x| \leqslant \frac{T_{p e r, B}}{2}
$$

(5) 正四方形相对边对基准 $\mathrm{B}$ 的平行度。

公差要求为 $T_{p a r}=0.013$ 。转化为直线所在平面 对于基准平面的平面度问题进行求解。

$$
|\Delta y| \leqslant \frac{T_{p a r}}{2}
$$


(6) 菱形边的直线度。

公差要求为 $T_{s t r, \text { rho }}=0.010$ 。由于菱形边高度尺 寸较小, 故加工时刀具转角误差的影响可忽略, 该 问题转化为直线所在平面的平面度问题进行求解 (2.1.1(2)所述情况)。如图 10 所示, 设所分析直线的 方位角为 $\theta\left(\theta=30^{\circ}\right.$ 或 $\left.\theta=-60^{\circ}\right)$, 则其所在平面的 方程可以描述为

$$
\tan \theta \cdot x-y=0
$$

平面法向量 $\boldsymbol{n}=\left(\begin{array}{lll}\tan \theta & -1 & 0\end{array}\right)^{\mathrm{T}}$, 则利用前文结论有 独立约束条件为

$$
\left|\hat{\boldsymbol{n}}^{\mathrm{T}} \lambda\right| \leqslant \frac{T_{\text {str,rho }}}{2} \quad \lambda=\left(\begin{array}{c}
\Delta x \\
0 \\
0
\end{array}\right),\left(\begin{array}{c}
0 \\
\Delta y \\
0
\end{array}\right),\left(\begin{array}{c}
0 \\
0 \\
\Delta z
\end{array}\right)
$$

综合约束条件为

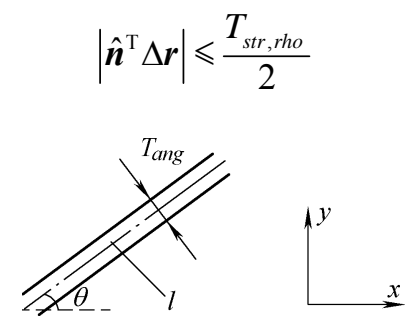

图 10 斜边直线度

（7）菱形四边对基准 $\mathrm{B}$ 的倾斜度。

公差要求为 $T_{a n g}=0.013$ 。由于 $T_{a n g} \geqslant T_{\text {str, rho }}$, 所 以只要公差项(6)的条件满足, 则(7)一定能满足, 故 不必考虑。

(8) 圆的圆度。

公差要求为 $T_{c i r}=0.016$ 。

独立约束条件为

$$
|\Delta x| \leqslant \frac{T_{c i r}}{2},|\Delta y| \leqslant \frac{T_{c i r}}{2}
$$

综合约束条件为

$$
\sqrt{\Delta x^{2}+\Delta y^{2}} \leqslant \frac{T_{\text {cir }}}{2}
$$

(9) 外圆和中心孔的同心度。

公差要求为 $T_{c o n, 1}=0.016$ 。

若公差项(1)和(8)都满足, 则(9)一定满足, 故不 必考虑。

(10) 斜面的直线度。

公差要求为 $T_{s t r}=0.010$ 。

(11) 斜面对基准 $\mathrm{B}$ 的倾斜度。

公差要求为 $T_{a n g}=0.013$ 。

公差项(10)和(11)主要检测在某一轴线进给率 非常低时的两轴线性插补精度, 当只考虑机床几何 精度时, 可忽略不计。
(12) 镗孔相对于中心孔 $\mathrm{C}$ 的位置度。

公差要求为 $T_{p o s}=0.030$ 。如图 $11 \mathrm{a}$ 所示, $C$ 为 中心孔理想位置, $O$ 为镗孔中心理想位置, 由于中 心孔存在圆柱度误差, $O$ 点会有一个直径为 $T_{c y l}=0.01$ 的圆形变动范围, 假设 $O^{\prime}$ 点位于该范围 边界上任一点, 则为了满足 $T_{p o s}=0.030$ 的要求, 镗 孔中心必须落在以 $O^{\prime}$ 点为圆心、 $T_{p o s}$ 为直径的圆(虚 线圆)内。对边界上所有点执行上述操作, 并对镗孔 中心点的可能位置取交集, 得到图 $11 \mathrm{~b}$ 中的直径为 0.02 的中心粗虚线区域, 即只要镗孔中心落在该区 域内, 则一定满足位置度 $T_{p o s}=0.030$ 的要求。从而 可以推出镗孔的圆度必须满足 $T_{c i r, 2} \leqslant 0.020$ 。由于公 差项(8)中要求圆度误差小于等于 0.016 , 因而此处 镗孔的圆度误差也必定小于等于 0.016 , 故公差项 (12)的要求自然满足, 无需再考虑。

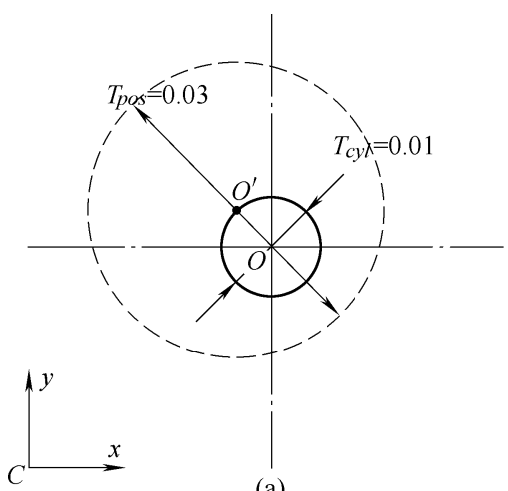

(a)

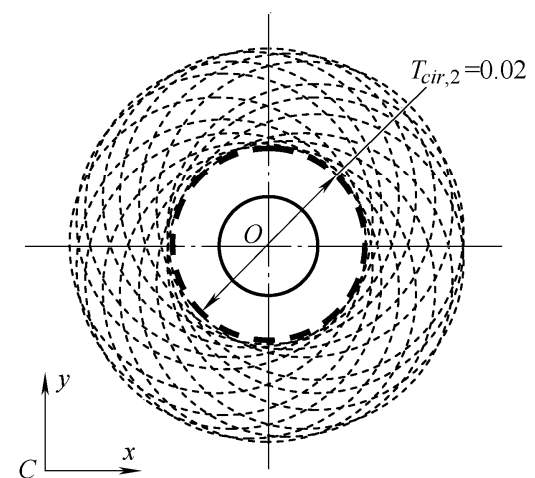

(b)

图 11 镗孔相对于中心孔的位置度

(13) 镗孔的内孔与外孔 D 的同心度。

公差要求为 $T_{c o n, 2}=0.013$ 。假设内外孔圆度公差 相同, 由 $T_{c o n, 2}=0.013$ 推出两者的圆度公差值应为 0.013 。前面的公差项(8)圆度为 0.016 , 由于大圆尺 寸远大于镗孔尺寸, 故其圆度理应略大于镗孔, 因 而认为镗孔的 0.013 的公差是可以满足的, 所以不 必考虑。

\section{2 综合公差分析求解}

所有公差项对应的约束条件如表 1 所示。假设 机床刀具相对工件间的三向位置误差与三向转角误 
差的设计变动范围分别相等, 即 $\Delta x 、 \Delta y 、 \Delta z$ 以及 $\Delta \alpha 、 \Delta \beta 、 \Delta \gamma$ 的变动范围一致, 对所有的约束条 件求交集可以得到一系列解集。取整数解以及半整 数解后可以得到 7 组解集(如表 2 所示)。

表 1 轮廓加工试件所有公差项对应的约束条件

\begin{tabular}{|c|c|c|c|}
\hline \multirow{3}{*}{$\begin{array}{l}\text { 公 } \\
\text { 差 }\end{array}$} & \multicolumn{2}{|c|}{ 独立约束条件 } & \multirow{3}{*}{ 综合约束条件 } \\
\hline & $|\Delta x|$, & $|\Delta \alpha|$ & \\
\hline & $|\Delta y|$ & $|\Delta \beta|$ & \\
\hline (1) & $\leqslant 5$ & $\leqslant 62.5$ & $(0.08 \Delta \beta+\Delta x)^{2}+(0.08 \Delta \alpha+\Delta y)^{2} \leqslant 5^{2}$ \\
\hline$(2)$ & $\leqslant 5$ & $\leqslant 62.5$ & $(0.08 \Delta \beta+\Delta x)^{2}+(0.08 \Delta \alpha+\Delta y)^{2} \leqslant 5^{2}$ \\
\hline (3) & $\leqslant 5$ & - & - \\
\hline (4) & $\leqslant 6.5$ & - & - \\
\hline$(5)$ & $\leqslant 6.5$ & - & - \\
\hline$(6)$ & $\leqslant 5.8$ & - & $|\Delta x-\sqrt{3} \Delta y| \leqslant 10,|\sqrt{3} \Delta x+\Delta y| \leqslant 10$ \\
\hline (7) & - & - & (6)满足, 则(7)满足 \\
\hline$(8)$ & $\leqslant 8$ & - & $\Delta x^{2}+\Delta y^{2} \mid \leqslant 8^{2}$ \\
\hline (9) & - & - & (1)、(8)满足, 则(9)满足 \\
\hline (10) & - & - & - \\
\hline$(11)$ & 一 & - & - \\
\hline$(12)$ & 一 & - & (8)满足, 则(12)满足 \\
\hline (13) & - & - & (8)满足, 则(13)满足 \\
\hline
\end{tabular}

单位: 位置误差 $\mu \mathrm{m}$, 转角误差 $\mu \mathrm{m} / \mathrm{m}$ 。

表 2 刀具相对工件间六维位姿误差变动范围

\begin{tabular}{ccc}
\hline 序号 & $|\Delta x| 、|\Delta y|$ 的最大值 & $|\Delta \alpha| 、|\Delta \beta|$ 的最大值 \\
\hline$(1)$ & $3.5 \mu \mathrm{m}$ & $0.5 \mu \mathrm{m} / \mathrm{m}$ \\
$(2)$ & $3.0 \mu \mathrm{m}$ & $6.5 \mu \mathrm{m} / \mathrm{m}$ \\
$(3)$ & $2.5 \mu \mathrm{m}$ & $13.0 \mu \mathrm{m} / \mathrm{m}$ \\
$(4)$ & $2.0 \mu \mathrm{m}$ & $19.0 \mu \mathrm{m} / \mathrm{m}$ \\
$(5)$ & $1.5 \mu \mathrm{m}$ & $25.5 \mu \mathrm{m} / \mathrm{m}$ \\
$(6)$ & $1.0 \mu \mathrm{m}$ & $31.5 \mu \mathrm{m} / \mathrm{m}$ \\
$(7)$ & $0.5 \mu \mathrm{m}$ & $38.0 \mu \mathrm{m} / \mathrm{m}$ \\
\hline
\end{tabular}

以第(2)组解集为例, 即只要 $|\Delta x|,|\Delta y| \leqslant 3 \mu \mathrm{m}$ 、 $|\Delta \alpha|,|\Delta \beta| \leqslant 6.5 \mu \mathrm{m} / \mathrm{m}$ 同时满足, 则试件的所有公 差项均可被满足。

需要指出的是以下几点。

(1) 最终选取哪一组解集需要结合机床的结构 特点以及应用要求做综合分析后决定;

(2) 若 $\Delta x 、 \Delta y 、 \Delta z$ 以及 $\Delta \alpha 、 \Delta \beta 、 \Delta \gamma$ 的变 动范围不一定一致, 此时不等式的解集为一系列多 维空间中的 “长方体”。

由上述分析过程可以得到试件各公差项的严格 程度以及受位置误差、转角误差的影响程度的大小。 例如, 公差项(1)、(2)对于 $\$$ 的允许变动范围的要求 等价且最为严格; 公差项(7)、(9)、(11)、(12)较为 宽松, 在其他公差项得到满足的前提下可自然被满 足; 公差项(3)至(6)、(8)仅与 $\$$ 中的位置误差有关。 此外也可以看出, 现有轮廓加工试件无法反应刀具
工件间 $z$ 向误差。

\section{4 结论}

本文针对机床精度设计过程中整机设计指标难 于合理确定的问题, 研究了基于待加工试件公差指 标反演机床刀具工件间相对位姿误差允许变动范围 的方法, 并以机床精度检验标准中轮廓加工试件为 例阐述了所提方法的应用流程。

该方法除了可以用于机床精度设计指标的确定 以外, 还可以用于: (1) 判断一个试件应当使用何种 精度等级的机床来加工, 或者判断现有机床能否加 工出合格的试件; (2) 分析试件各项公差的严格程 度, 以便合理安排加工工艺流程。该方法由于采用 极值约束条件, 因而求解得到的工具工件间相对位 姿误差的变动范围较为严格, 在实际应用中可依据 实际情况酶情放宽设计指标。

\section{参 考 文 献}

[1] SCHELLEKENS P, ROSIELLE N, VERMEULEN H, et al. Design for precision: current status and trends[J]. CIRP Annals-Manufacturing Technology， 1998，47(2): 557-586.

[2] TIAN W, GAO W, ZHANG D, et al. A general approach for error modeling of machine tools[J]. International Journal of Machine Tools and Manufacture, 2014, 79: $17-23$.

[3] 洪军, 郭俊康, 刘志刚, 等. 基于状态空间模型的精密 机床装配精度预测与调整工艺 $[\mathrm{J}]$. 机械工程学报, 2013, 49(6): 114-121.

HONG Jun, GUO Junkang, LIU Zhigang, et al. Assembly accuracy prediction and adjustment process modeling of precision machine tool based on state space model[J]. Journal of Mechanical Engineering, 2013, 49(6): 114-121.

[4] PRABHAHARAN G, ASOKAN P, RAMESH P, et al. Genetic-algorithm-based optimal tolerance allocation using a least-cost model[J]. The International Journal of Advanced Manufacturing Technology, 2004, 24(9): 647-660.

[5] HUANG T, CHETWYND D G, MEI J, et al. Tolerance design of a 2-DOF overconstrained translational parallel robot[J]. IEEE Transactions on Robotics, 2006, 22(1): 167-172.

[6] 刘鹏, 洪军, 刘志刚, 等. 采用自适应遗传算法的机床 公差分配研究 [J]. 西安交通大学学报, 2016, 50(1): 115-123.

LIU Peng, HONG Jun, LIU Zhigang, et al. Research on the tolerance allocation of machine tools based on 
adaptive genetic algorithm[J]. Journal of Xian Jiaotong University, 2016, 50(1): 115-123.

[7] DING W, ZHOU M, HUANG X, et al. Study on accuracy design of multi-axis machine tools oriented to remanufacturing[J]. Journal of Basic Science and Engineering, 2007, 15(4): 559-568.

[8] JI S, LI X, CAI H. Optimal tolerance allocation based on fuzzy comprehensive evaluation and genetic algorithm[J]. International Journal of Advanced Manufacturing Technology, 2000, 16: 461-468.

[9] HAQ A N, SIVAKUMAR K, SARAVANAN R, et al. Tolerance design optimization of machine elements using genetic algorithm[J]. International Journal of Advanced Manufacturing Technology, 2005, 25: 385-391.

[10］中华人民共和国国家质量监督检验检疫总局, 中国国 家标准化管理委员会. GB/T 20957.7-2007 精密加工 中心检验条件第 7 部分: 精加工试件精度检验[S]. 北
京: 中国标准出版社, 2007.

General Administration of Quality Supervision, Inspection and Quarantine of the People's Republic of China, Standardization Administration of the People's Republic of China. GB/T 20957.7-2007 The conditions for precision machining centres - Part 7: Accuracy of a finished test piece[S]. Beijing: Standards Press of China, 2007.

作者简介: 王丽娜, 女, 1988 年出生, 讲师。主要研究方向为制造技术。 E-mail: wanglina_0718@126.com

田文杰(通信作者), 男, 1986 年出生, 博士, 讲师。主要研究方向为数 控机床与工业机器人的误差建模、检测与补偿等。

E-mail: wenjietian@tju.edu.cn

张大卫, 男, 1962 年出生, 博士, 教授, 博士研究生导师。主要研究方 向为机械系统动力学、高速精密定位技术、高速切削理论与应用等。

E-mail: medzhang@tju.edu.cn

高卫国, 男, 1974 年出生, 博士, 副教授, 博士研究生导师。主要研究 方向为智能机床设计与制造关键技术等。

E-mail: gaowg@tju.edu.cn 